\title{
Dijkstra algorithm for shortest path problem under interval-valued Pythagorean fuzzy environment
}

\author{
Mohammad Enayattabar $^{1} \cdot$ Ali Ebrahimnejad $^{2}$ (D) $\cdot$ Homayun Motameni $^{3}$
}

Received: 31 August 2018 / Accepted: 5 October 2018 / Published online: 25 October 2018

(c) The Author(s) 2018

\begin{abstract}
Pythagorean fuzzy set as an extension of fuzzy set has been presented to handle the uncertainty in real-world decision-making problems. In this work, we formulate a shortest path (SP) problem in an interval-valued Pythagorean fuzzy environment. Here, the costs related to arcs are taken in the form of interval-valued Pythagorean fuzzy numbers (IVPFNs). The main contributions of this paper are fourfold: (1) the interval-valued Pythagorean fuzzy optimality conditions in directed networks are described to design of solution algorithm. (2) To do this, an improved score function is used to compare the costs between different paths with their arc costs represented by IVPFNs. (3) Based on these optimality conditions and the improved score function, the traditional Dijkstra algorithm is extended to find the cost of interval-valued Pythagorean fuzzy SP (IVPFSP) and corresponding IVPFSP. (4) Finally, a small sized telecommunication network is provided to illustrate the potential application of the proposed method.
\end{abstract}

Keywords Shortest path problem $\cdot$ Pythagorean fuzzy numbers $\cdot$ Score function $\cdot$ Dijkstra algorithm

\section{Introduction}

Shortest path (SP) problems lie at the heart of network flows. They arise frequently in practice since the aim of a wide variety of real-life problems is to send some goods between two specified nodes in a network as cheaply as possible. Therefore, SP problems with the aim of finding a path with the least cost (time or length) from the source node to the destination node can be used for formulating such real applications. Traditionally, it has been generally assumed that traversal costs of arcs are expressed in terms of crisp numbers. But, these values are generally imprecise or vague in nature since the costs

\section{Ali Ebrahimnejad}

aemarzoun@gmail.com; a.ebrahimnejad@qaemiau.ac.ir

Mohammad Enayattabar

m.enayat@gmail.com

Homayun Motameni

motameni@iausari.ac.ir

1 Department of Computer Engineering, Babol Branch, Islamic Azad University, Babol, Iran

2 Department of Mathematics, Qaemshahr Branch, Islamic Azad University, Qaemshahr, Iran

3 Department of Computer Engineering, Sari Branch, Islamic Azad Univeristy, Sari, Iran fluctuate with traffic conditions and weather. For this, Zadeh [1] proposed the fuzzy set theory which is a very useful tool to cope with imprecise data in SP problems. Consequently, various attempts have been made by researchers for different types of SP problems in fuzzy environment.

Based on possibility theory, Okada [2] proposed an algorithm for solving fuzzy SP problem to determine the degree of possibility for each arc. Keshavarz and Khorram [3] simplified the fuzzy SP problem into a bi-level programming problem and proposed an efficient algorithm, based on the parametric SP problem for solving the resulting problem. Dou et al. [4] solved the fuzzy SP problem in multiple constraints network with vague multi-criteria decision making methods based on similarity measures. Deng et al. [5] extended the Dijkstra algorithm for solving fuzzy SP problems using the graded mean integration representation of fuzzy numbers. Moreover, some authors [6, 7] focused on computing a shortest path in the network having various types of fuzzy arc cost based on heuristic algorithms.

However, fuzzy set takes only a membership function and cannot express non-membership function. Here, the degree of non-membership is just the complement of the degree of membership. Then Atanassov [8] introduced intuitionistic FS (IFS) to incorporate the non-membership degree during the analysis. Here, the sum of membership degree and the non- 
membership degree is equal to or less than one. Under IFS environment, some researchers pay more attention to solving SP problem with intuitionistic fuzzy arc costs. Mukherjee [9] considered the SP problem in an intuitionistic fuzzy environment. Geetharamani and Jayagowri [10] proposed a new algorithm to deal with the IFSP problem using intuitionistic fuzzy shortest path length procedure and similarity measure. Biswas et al. [11] developed a method to search for an intuitionistic fuzzy shortest path between the source node and the destination node. Kumar et al. [12] proposed an algorithm to find the shortest path and shortest distance in a network where interval-valued fuzzy intuitionistic arc weights. Sujatha and Hyacinta [13] proposed two different approaches for solving the SP problem under intuitionistic environment. Motameni and Ebrahimnejad [14] worked on solving SP with an additional constraint under intuitionistic fuzzy environment.

However, there may be a situation where the sum of the membership and non-membership degrees is greater than one. Thus, Yager [15] introduced a generalization of IFS called Pythagorean fuzzy set (PFS) where the square sum of the membership and non-membership degrees sum is equal to or less than one. Zhang [16] extended the concept of PFSs to interval-valued PF (IVPF) sets where the values of membership and non-membership functions are expressed in terms of intervals rather than crisp numbers. After PFSs, IVPF set and different extensions of them have been used by some researchers to process fuzzy information in multi criteria decision making problems [17-27]. However, to the best of our knowledge there is no study in the literature on solving SP problems in IVPF environment.

Thus, the object of this paper was to propose a method for solving SP problems under IVPF environment. To do this, we first present the mathematical formulation on SP problems where the traversal costs of arcs are represented in terms of interval-valued Pythagorean fuzzy numbers (IVPFNs). Then, we describe the optimality conditions in IVPF networks to design of solution algorithm. To do this, an improved score function is used to compare the costs between different paths with their arc costs represented by IVPFNs. Then, the traditional Dijkstra algorithm is extended to find the cost of interval-valued Pythagorean fuzzy SP (IVPFSP) and corresponding IVPFSP. The proposed algorithm is illustrated by a small sized telecommunication network under IVPF environment.

The rest of the paper is organized as follows: In Sect. 2, some basic concepts of interval-valued Pythagorean fuzzy sets are presented. In Sect. 3, the mathematical formulation of the SP problem under IVPF environment is given. The IVPF shortest path optimality conditions and the extended Dijkstra's algorithm are presented Sect. 4. In Sect. 5, a numerical example is given to illustrate the proposed solution technique. Last, the paper is concluded in Sect. 6.

\section{Preliminaries}

In this section, we present some necessary background and notions of the interval-valued Pythagorean fuzzy numbers which are applied throughout this paper [8, 15, 17-19].

Definition 1 Let $X$ denotes the universe set. An intuitionistic fuzzy set (IFS) $\tilde{A}^{I}$ in $X$ is defined by a set of ordered triple $\tilde{A}^{I}=\left\{\left\langle x, \mu_{\tilde{A}^{I}}(x), v_{\tilde{A}^{I}}(x)\right\rangle ; x \in X\right\}$, where the functions $\mu_{\tilde{A}^{I}}(x): X \rightarrow[0,1]$ and $v_{\tilde{A}^{I}}(x): X \rightarrow[0$, 1], respectively, represent the membership degree and nonmembership degree of $x$ in $\tilde{A}^{I}$ such that for each element $x \in X, 0 \leq \mu_{\tilde{A}^{I}}(x)+v_{\tilde{A}^{I}}(x) \leq 1$. For any IFS $\tilde{A}^{I}$ and $x \in X, \pi_{\tilde{A}^{I}}(x)=1-\mu_{\tilde{A}^{I}}(x)-v_{\tilde{A}^{I}}(x)$ is said to be the degree of hesitation of $x$ to $\tilde{A}^{I}$.

Definition 2 A Pythagorean fuzzy set (PFS) $\tilde{A}^{P}$ in the universe set $X$ is defined by the set $\tilde{A}^{P}=$ $\left\{\left\langle x, \mu_{\tilde{A}^{P}}(x), v_{\tilde{A}^{P}}(x)\right| ; x \in X\right\}$, where the membership function $\mu_{\tilde{A}^{P}}(x): X \rightarrow[0,1]$ and non-membership function $v_{\tilde{A}^{P}}(x): X \rightarrow[0,1]$, satisfy the condition $\left[\mu_{\tilde{A}^{P}}(x)\right]^{2}+$ $\left[v_{\tilde{A}^{P}}(x)\right]^{2} \leq 1$ for each element $x \in X$. For any PFS $\tilde{A}^{P}$ and $x \in X, \pi_{\tilde{A}^{P}}(x)=\sqrt{1-\left[\mu_{\tilde{A}^{P}}(x)\right]^{2}-\left[v_{\tilde{A}^{P}}(x)\right]^{2}}$ is said to be the degree of hesitation of $x$ to $\tilde{A}^{P}$.

For convenience, Zhang and $\mathrm{Xu}$ [16] called $\left(\mu_{\tilde{A}^{P}}(x), v_{\tilde{A}^{P}}(x)\right)$ a Pythagorean fuzzy number $(\mathrm{PFN})$ denoted by $\tilde{A}^{P}=\left(\mu_{\tilde{A}^{P}}, v_{\tilde{A}^{P}}\right)$.

For example, assume that the membership degree of an element to a fuzzy set is $\frac{\sqrt{3}}{2}$ and the non-membership degree of this element is $\frac{1}{2}$. This situation cannot be described by utilizing the IFS since $\frac{\sqrt{3}}{2}+\frac{1}{2}>1$. However, $\left(\frac{\sqrt{3}}{2}\right)^{2}+\left(\frac{1}{2}\right)^{2} \leq$ 1 ; thus PFN is suitable to handle this situation.

Definition 3 The score function and the accuracy function of any PFN $\tilde{A}^{P}=\left(\mu_{\tilde{A}^{P}}, v_{\tilde{A}^{P}}\right)$ are defined as follows, respectively:

$S\left(\tilde{A}^{P}\right)=\left(\mu_{\tilde{A}^{P}}\right)^{2}-\left(v_{\tilde{A}^{P}}\right)^{2}, \quad S\left(\tilde{A}^{P}\right) \in[-1,1]$

$H\left(\tilde{A}^{P}\right)=\left(\mu_{\tilde{A}^{P}}\right)^{2}+\left(v_{\tilde{A}^{P}}\right)^{2}, \quad H\left(\tilde{A}^{P}\right) \in[0,1]$

Definition 4 An interval-valued Pythagorean fuzzy set (IVPFS) $\underline{\tilde{A}}^{P}$ in the universe set $X$ is defined by the set $\underline{\tilde{A}}^{P}=\left\{\left\langle x, \underline{-}_{\underline{\tilde{\tilde{A}}}} P(x), \underline{\bar{v}}_{\underline{\tilde{\tilde{A}}}^{P}}(x)\right\rangle ; x \in X\right\}$ where $\underline{-}_{\underline{\tilde{\tilde{A}}}} P(x)=\left[\underline{\underline{\mu}}_{\underline{\tilde{\tilde{A}}}} P(x), \bar{\mu}_{\underline{\tilde{\tilde{A}}}} P(x)\right] \subseteq[0,1]$ and $\underline{\bar{v}}_{\underline{\tilde{\tilde{A}}}^{P}}(x)=$ $\left[\underline{v}_{\underline{\tilde{\tilde{A}}}} P(x), \bar{v}_{\overline{\tilde{A}}^{P}}(x)\right] \subseteq[0,1]$ are inteval numbers satisfying the condition $\left[\bar{\mu}_{\underline{\tilde{\tilde{A}}}} P(x)\right]^{2}+\left[\bar{v}_{\underline{\tilde{A}}} P(x)\right]^{2} \leq$ 
1 for each element $x \in X$. For any IVPFS $\underline{\tilde{\tilde{A}}}^{P}$ and $x \in X, \underline{\bar{\pi}}_{\tilde{\tilde{\tilde{A}}} P}(x)=\left[_{\underline{\tilde{\tilde{A}}}^{P}}(x), \overline{\bar{\pi}}_{\tilde{\tilde{A}}^{P}}(x)\right]=$

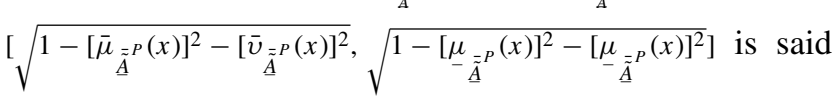
to be the hesitation interval of $x$ to $\overline{\tilde{A}}^{P}$.

For an IVPFS $\underline{\tilde{\tilde{A}}}^{P}$, the pair

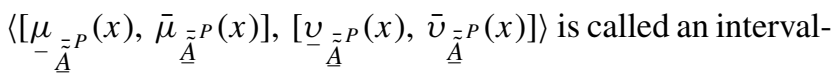
valued Pythagorean fuzzy number (IVPFN) denoted by

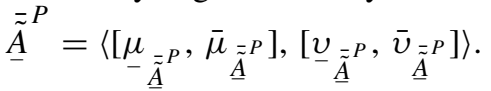

Remark 1 [18] Two IVPFNs, $\underline{\tilde{0}}^{P}=\langle[0,0],[1,1]\rangle$ and $\underline{\tilde{1}}^{P}=\langle[1,1],[0,0]\rangle$ are the smallest and the largest IVPFNs, respectively.

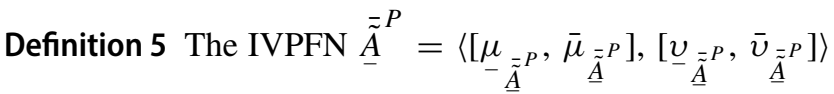
is siad to be non-negative if $\underline{\underline{\mu}}_{\underline{\tilde{\tilde{A}}}} P \geq \underline{\underline{\tilde{A}}}^{P}$ and $\bar{\mu}_{\underline{\tilde{\tilde{A}}}} P \geq \bar{v}_{\overline{\tilde{A}}^{P}}$.
Definition 7 [19] The score function and the accuracy func-

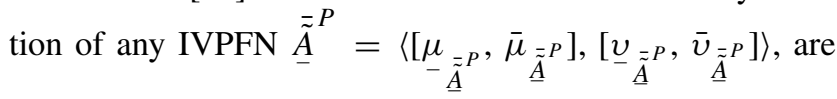
defined as follows, respectively:

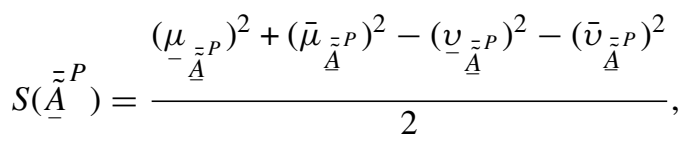

$$
S\left(\underline{\tilde{\tilde{A}}}^{P}\right) \in[-1,1]
$$

$$
H\left(\underline{\tilde{\tilde{A}}}^{P}\right)=\frac{\left(\underline{-}^{\tilde{\tilde{A}} P}\right)^{2}+\left(\bar{\mu}_{\underline{\tilde{\tilde{A}}}^{P}}\right)^{2}+\left(\underline{v}_{\underline{\tilde{\tilde{A}}}^{P}}\right)^{2}+\left(\overline{\bar{v}}_{\left.\underline{\tilde{\tilde{A}}}^{P}\right)^{2}}\right.}{H\left(\underline{\tilde{\tilde{A}}}^{P}\right) \in[0,1]},
$$

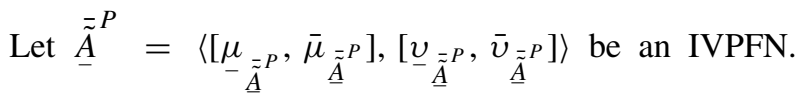
Based on three concepts, hesitation interval index, favor degrees and against degrees relative to $\underline{\tilde{A}}^{P}$, Garg [19] defined an improved score function of $\overline{\tilde{A}}^{P}$ as follows:

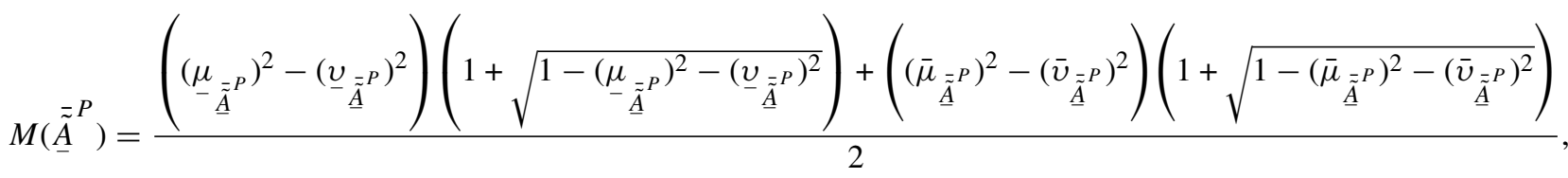

where $M\left(\underline{\tilde{\tilde{A}}}^{P}\right) \in[-1,1]$.

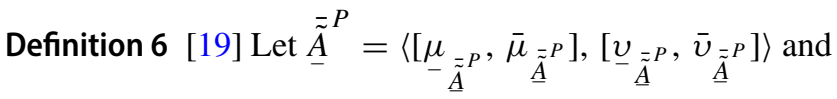

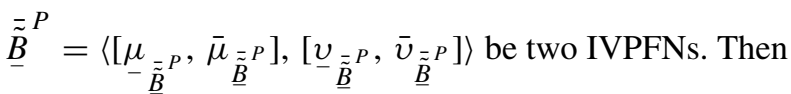

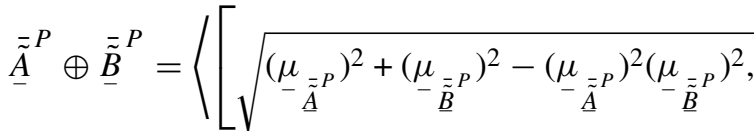

$$
\begin{aligned}
& \left.\times \sqrt{\left(\bar{\mu}_{\tilde{\tilde{\tilde{A}}}} P\right)^{2}+\left(\bar{\mu}_{\tilde{\tilde{\tilde{B}}}} P\right)^{2}-\left(\bar{\mu}_{\tilde{\tilde{A}}} P\right)^{2}\left(\bar{\mu}_{\underline{\tilde{\tilde{B}}}} P\right)^{2}}\right],
\end{aligned}
$$

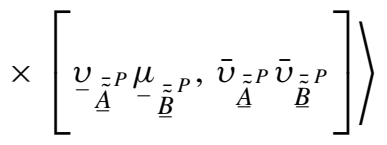

\section{Remark 2 For any IVPFN $\underline{\tilde{\tilde{A}}}^{P}=$}

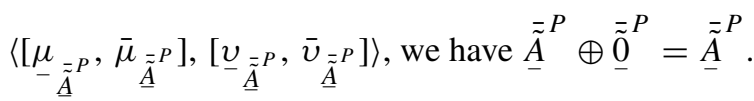

Remark 3 [19] For two IVPFNs $\underline{\tilde{\tilde{0}}}^{P}=\langle[0,0],[1,1]\rangle$ and $\underline{\tilde{\tilde{1}}}^{P}=\langle[1,1],[0,0]\rangle$, we have $M\left(\underline{\tilde{\tilde{0}}}^{P}\right)=-1$ and $M\left(\underline{\tilde{\tilde{1}}}^{P}\right)=$ 1.

Proposition 1 If $\underline{\tilde{\tilde{A}}}^{P}=\left\langle\left[\mu_{-\overline{\tilde{A}}^{P}}, \bar{\mu}_{\underline{\tilde{\tilde{A}}}}\right],\left[\underline{\underline{v}}_{\underline{\tilde{A}}^{P}}, \bar{v}_{\underline{\tilde{\tilde{A}}}} P\right]\right\rangle$ be a nonnegative IVPFN, then $M\left(\overline{\tilde{A}}^{P}\right) \geq 0$.

Proof According to Definition 5, we have $\underline{-}_{\overline{\tilde{A}}^{P}} \geq \underline{\underline{v}}_{\overline{\tilde{A}}^{P}}$ and $\bar{\mu}_{\underline{\tilde{\tilde{A}}}} P \geq \bar{v}_{\overline{\tilde{A}}^{\tilde{A}} P \text {. Therefore, }}\left(\underline{\mu}_{-\underline{\tilde{\tilde{A}}}}\right)^{2}-\left(\underline{v}_{\underline{\tilde{\tilde{A}}}} P\right)^{2} \geq 0$ and $\left(\bar{\mu}_{\tilde{\tilde{\tilde{A}}}}\right)^{2}-\left(\bar{v}_{\overline{\tilde{A}}^{P}}\right)^{2} \geq 0$. Now, based on Eq. (1), we conclude that $M\left(\overline{\tilde{A}}^{P}\right) \geq 0$.

Remark 4 Garg [19] used the improved score function given in (1) to compare two IVPFNs 


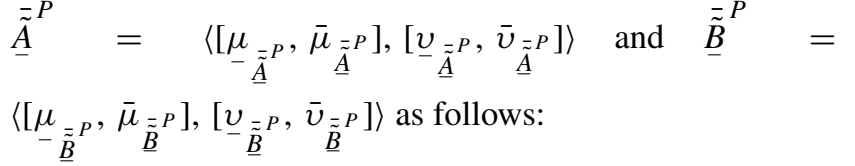

- If $M\left(\underline{\tilde{\tilde{A}}}^{P}\right)>M\left(\underline{\tilde{\tilde{B}}}^{P}\right)$, then $\underline{\tilde{\tilde{A}}}^{P} \succ \underline{\tilde{\tilde{B}}}^{P}$.

- If $M\left(\underline{\tilde{\tilde{A}}}^{P}\right)=M\left(\underline{\tilde{\tilde{B}}}^{P}\right)$, then $\underline{\tilde{\tilde{A}}}^{P} \sim \underline{\tilde{\tilde{B}}}^{P}$.

The existing approaches for comparing two IVPFNs are based on the score and accuracy functions. Such approaches neglect the hesitation interval index and thus they are unable to give the exact position of IVPFNs. However, the ranking function given in (1) overcomes this shortcoming and provides exact positions of IVPFNs by sufficiently considering the indeterminacy information of an IVPFS.

\section{Interval-valued Fuzzy Pythagorean Shortest Path problem}

In this section, the mathematical formulation of the intervalvalued Pythagorean fuzzy shortest path (IVPFSP) problem is presented.

We consider a directed network $G=(V, E)$, with node set $V=\{1,2, \ldots, m\}$ and arc set $E=\{(i, j): i, j \in V, i \neq j\}$. For two different nodes $i, j \in E$, the ordered pair $(i, j)$ denotes an arc of the network. Two nodes 1 and $m$ are considered as the source and destination nodes of the network, respectively. It is supposed that there is only one directed arc $(i, j)$ from node $i$ to node $j$. A path $p_{i j}$ from node $i$ to node $j$ is a sequence of arcs $p_{i j}=\left\{\left(i, i_{1}\right),\left(i_{1}, i_{2}\right), \ldots,\left(i_{k}, j\right)\right\}$ in which the initial node of each arc is same as the terminal node of preceding arc in the sequence. The cost of a directed path is defined as the sum of the arc costs the path. We assume the network contains a directed path from the source node to every other node in the network.

The non-negative weight $c_{i j}$ is associated with each arc $(i, j)$ representing the cost associated with the respective arc. The main purpose of the SP problem is to find a path with the least cost, from node 1 to node $m$. Conventional SP problems consider certain and precise values for the arc costs, which is not always the case in real-life problems. As time and cost fluctuate with traffic conditions, weather and payload, different extensions of fuzzy set can be utilized to represent imprecise and vague arc costs. In this work, interval-valued Pythagorean fuzzy numbers are used to represent the vague parameters of the SP problem under consideration. The resulting problem is, therefore, referred to as an interval-valued Pythagorean fuzzy SP (IVPFSP) problem.

An IVPFSP problem having non-negative IVPFNs for the arc costs is formulated as follows:

$$
\begin{aligned}
& \min \underline{\tilde{Z}}=\sum_{i=1}^{m} \sum_{j=1}^{m} \underline{\tilde{\bar{c}}}_{i j}^{P} x_{i j} \\
& \text { s.t. } \sum_{j=1}^{n} x_{i j}-\sum_{k=1}^{m} x_{k i}= \begin{cases}1, & i=1, \\
0, & i \neq 1, m, \\
-1, & i=m,\end{cases} \\
& \quad x_{i j} \geq 0, \quad i, \quad j=1,2, \ldots, m .
\end{aligned}
$$

If $\operatorname{arc}(i, j)$ is in the path, then $x_{i j}=1$; otherwise $x_{i j}=0$.

Let $T_{s t}$ denote the set of all paths from node $s$ to node $t$. Define $\overline{\tilde{C}}^{P}\left(p_{u v}\right)=\sum_{(i, j) \in p_{u v}} \underline{\tilde{\tilde{C}}}_{i j}^{P}$ as the interval-valued Pythagorean fuzzy cost of path $p_{u v}$ from node $u$ to node $v$.

\section{Extended Dijkstra Algorithm in IVPF environment}

In this section, the traditional Dijkstra algorithm is extended for solving the IVPFSP problem (2).

Theorem 1 (IVPFSP optimality conditions) For every node $j \in E$, let $\underline{\tilde{\tilde{w}}}_{j}^{P}$ denote the interval-valued Pythagorean fuzzy cost of some directed path from the source node 1 to node $j$. Then, IVPFNs $\underline{\tilde{\tilde{w}}}_{j}^{P}$ represent IVPFSP costs if and only if they satisfy the following IVPFSP optimality conditions:

$\overline{\tilde{\tilde{w}}}_{j}^{P} \preceq \underline{\tilde{\tilde{w}}}_{i}^{P}+\underline{\tilde{\bar{c}}}_{i j}^{P}$ for all $(i, j) \in E$

Proof If $\underline{\tilde{w}}_{j}^{P}$ is the IVPF cost of a shortest path from the source node 1 to node $j$, then it must satisfy the conditions (3). Suppose not, i.e. $\underline{\tilde{\tilde{w}}}_{j}^{P} \succ \underline{\tilde{\tilde{w}}}_{i}{ }_{i}+\underline{\tilde{\bar{c}}}_{i j}^{P}$ for some $\operatorname{arc}(i, j) \in E$. By assumption $\underline{\tilde{w}}_{i}^{P}$ is the IVPF cost of a directed path like $p_{1 i}$ from the source node 1 to node $i$. This path plus the arc $(i, j)$ constructs a new path from the source node 1 to node $j$ with the IVPF cost $\underline{\tilde{\tilde{w}}}_{i}{ }_{i}+\underline{\tilde{\bar{c}}}_{i j}^{P}$. This contradicts the optimality of IVPFSP cost $\underline{\tilde{w}}_{j}^{P}$.

We now show if the IVPF costs $\underline{\tilde{w}}_{j}^{P}$ satisfy the conditions in (3), they represent IVPFSP costs. To do this, consider any IVPF costs $\underline{\tilde{w}}^{P}$ satisfying (3). Let $1=i_{1}-i_{2}-\cdots-i_{k}=j$ be any path $p_{1 j}$ from the source node 1 to node $j$. The conditions (3) imply that

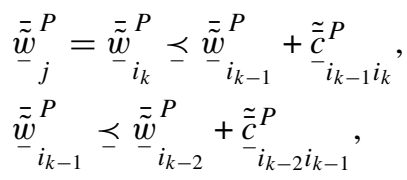


$\underline{\tilde{\tilde{w}}}_{i_{2}}^{P} \preceq \underline{\tilde{\tilde{w}}}_{i_{1}}^{P}+\underline{\bar{c}}_{i_{1} i_{2}}^{P}=\underline{\bar{c}}_{i_{1} i_{2}}^{P}$,

The last equality follows from the fact that $\underline{\tilde{\tilde{w}}}_{i_{1}}^{P}=\underline{\tilde{\tilde{w}}}_{1}^{P}=$ $\langle[0,0],[1,1]\rangle$. Adding the equalities, we find that

$$
\begin{aligned}
& \underline{\tilde{\tilde{w}}}_{j}^{P}=\underline{\tilde{\tilde{w}}}_{i_{k}}^{P} \preceq \underline{\underline{\bar{c}}}_{i_{k-1} i_{k}}+\underline{\overline{\bar{c}}}_{i_{k-2} i_{k-1}}+\cdots+\underline{\overline{\bar{c}}}_{{ }_{i_{1} i_{2}}} \\
& =\sum_{(i, j) \in p_{1 j}} \underline{\overline{\bar{c}}}^{P}=\overline{\tilde{C}}^{P}\left(p_{i j}\right)
\end{aligned}
$$

Thus $\underline{\tilde{\tilde{w}}}_{j}^{P}$ is a lower bound on the IVPF cost of any path from the source node 1 to node $j$. On the other hand, since $\overline{\tilde{w}}_{j}^{P}$ is the IVPF cost of some path from the source node 1 to node $j$, it also is an upper bound on the IVPFSP cost. Therefore, $\underline{\tilde{\tilde{w}}}_{j}^{P}$ is the IVPFSP cost.

Now, we are at a position to describe the extended Dijkstra's algorithm for finding IVPFSP from the source node 1 to destination node $m$ in a directed network with IVPF costs. This algorithm automatically yields the IVPFSP from the source node 1 to all other nodes as well.

\section{Algorithm 1: The extended Dijkstra's algorithm under IVPF environment}

Initialization step:

Set $\underline{\tilde{\tilde{w}}}_{1}^{P}=\underline{\tilde{\tilde{0}}}^{P}=\langle[0,0],[1,1]\rangle, S=\{1\}$ and Pred $\{1\}=0$.

Main step:

Let $\bar{S}=V-S$ and consider all arcs in the set $(S, \bar{S})=$ $\{(i, j): i \in S, j \in \bar{S}\}$. Let

$M\left(\underline{\tilde{\tilde{w}}}_{u}^{P} \oplus \underline{\overline{\bar{c}}}_{u v}^{P}\right)=\min \left\{M\left(\overline{\overline{\tilde{w}}}_{i}^{P}+\underline{\overline{\bar{c}}}_{i j} P\right) ;(i, j) \in(S, \bar{S})\right\}$

Set ${\overline{\tilde{w}}_{v}^{P}}_{v}^{P}=\underline{\overline{\tilde{w}}}_{u}^{P} \oplus \underline{\tilde{\bar{c}}}_{u v}^{P}, \operatorname{Pred}\{v\}=u$, and $S:=S \cup\{v\}$. Repeat the main step exactly $m-1$ times and then stop. The IVPF cost of the SP and the corresponding IVPFSP are at hand.

Remark 5 Note that Pred $\{i\}$ gives the predecessor node of node $i$.

Theorem 2 The extended Dijkstra's algorithm under IVPF environment yields the IVPFSP and its IVPS cost.

Proof Assume, inductively, that each $\underline{\tilde{\tilde{w}}}_{i}{ }_{i}$ for $i \in S$ represents the IVPF cost of the IVPFSP from the source node 1 to node $i$. This is true for $i=1$ since $\underline{\tilde{\tilde{w}}}_{1}^{P}=\underline{\tilde{\tilde{0}}}^{P}=\langle[0,0],[1,1]\rangle$.
Consider the point at which a new node $v$ is trying to be added to $S$. Assume that

$$
M\left(\overline{\tilde{\tilde{w}}}_{u}^{P} \oplus \underline{\tilde{\bar{c}}}_{u v} P\right)=\min \left\{M\left(\overline{\tilde{\tilde{w}}}_{i}{ }^{P}+\underline{\bar{c}}_{i j} P\right) ;(i, j) \in(S, \bar{S})\right\}
$$

We shall show that the IVPFSP from the source node 1 to node $v$ has the IVPF cost $\underline{\tilde{\tilde{w}}}_{v}^{P}=\underline{\overline{\tilde{w}}}_{u}^{P} \oplus \underline{\tilde{\bar{c}}}_{u v}^{P}$ and can be constructed iteratively as the IVPFSP from the source node 1 to node $u$ plus the arc $(u, v)$.Consider any IVPF path $p_{1 v}$ from the source node 1 to node $v$. It suffices to prove that $M\left(\sum_{(i, j) \in p_{1 v}} \underline{\tilde{\bar{c}}}_{i j}^{P}\right)=M\left(\underline{\tilde{\tilde{C}}}^{P}\left(p_{1 v}\right)\right) \geq M\left(\underline{\tilde{\tilde{w}}}_{v}{ }_{v}\right)$. Since $1 \in S$ and $v \in \bar{S}$, there exists an $\operatorname{arc}(i, j) \in p_{1 v}$ where $i \in S$ and $j \in \bar{S}$. Hence, path $p_{1 v}$ can be decomposed into three parts $p_{1 i},(i, j)$ and $p_{j v}$. Based on the induction hypothesis, the IVPF cost of $p_{1 i}$ is greater than or equal to $\underline{\tilde{\tilde{w}}}_{i}^{P}$, i.e. $M\left(\underline{\tilde{\tilde{C}}}^{P}\left(p_{1 i}\right)\right) \geq M\left(\underline{\tilde{\tilde{w}}}_{i}{ }^{P}\right)$. Since the IVPF costs of all arcs are assumed to be nonnegative, $M\left(\underline{\tilde{\tilde{C}}}^{P}\left(p_{j v}\right)\right) \geq 0$ (see Proposition 1). Thus, $M\left(\underline{\tilde{\tilde{C}}}^{P}\left(p_{1 v}\right)\right) \succeq M\left(\underline{\tilde{\tilde{w}}}_{i}^{P} \oplus \underline{\bar{c}}_{i j}^{P}\right)$. Based on Eq. (3) and since $M\left(\underline{\tilde{\tilde{w}}}_{v}^{P}\right)=M\left(\underline{\tilde{\tilde{w}}}_{u}^{P} \oplus \underline{\bar{c}}_{u v} P\right)$, we conclude that $M\left(\underline{\tilde{\tilde{C}}}^{P}\left(p_{1 v}\right)\right) \succeq M\left(\underline{\tilde{\tilde{w}}}_{v}^{P}\right)$. This completes the induction argument and the validity of the algorithm is established.

\section{Numerical example}

In this section, a small sized telecommunication network is provided to illustrate the potential application of the proposed method.

Consider a mobile service company which handles six geographical centers. A configuration of a telecommunication network is presented in Fig. 1. Assume that the cost between any two centers is an interval-valued Pythagorean fuzzy number (the arc costs are given in Table 1). The company wants to find an interval-valued Pythagorean fuzzy shortest path for an effective message flow amongst the centers.

The interval-valued Pythagorean fuzzy shortest path cost and the corresponding interval-valued Pythagorean fuzzy shortest path can be obtained using the proposed extended Dijkstra's algorithm (Algorithm 1), as follows:

Initialization step:

Set $\underline{\tilde{\tilde{w}}}^{P}=\overline{\tilde{0}}^{P}=\langle[0,0],[1,1]\rangle, S=\{1\}$ and Pred $\{1\}=0$. Main step: 


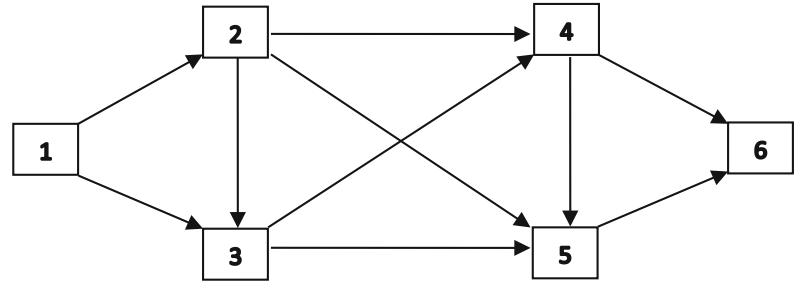

Fig. 1 An example of IVFSP network

Table 1 Arc information in terms of IVPFNs

\begin{tabular}{lc}
\hline Arc & Intuitionistic fuzzy cost \\
\hline$(1,2)$ & $\langle[0.4,0.5],[0.3,0.4]\rangle$ \\
$(1,3)$ & $\langle[0.6,0.7],[0.2,0.3]\rangle$ \\
$(2,3)$ & $\langle[0.3,0.6],[0.3,0.4]\rangle$ \\
$(2,4)$ & $\langle[0.7,0.8],[0.1,0.2]\rangle$ \\
$(2,5)$ & $\langle[0.6,0.7],[0.2,0.3]\rangle$ \\
$(3,4)$ & $\langle[0.4,0.6],[0.2,0.4]\rangle$ \\
$(3,5)$ & $\langle[0.7,0.8],[0.3,0.5]\rangle$ \\
$(4,5)$ & $\langle[0.5,0.6],[0.1,0.3]\rangle$ \\
$(4,6)$ & $\langle[0.4,0.7],[0.1,0.2]\rangle$ \\
$(5,6)$ & $\langle[0.3,0.4],[0.1,0.2]\rangle$ \\
\hline
\end{tabular}

Iteration 1:

Let $\bar{S}=V-S=\{2,3,4,5,6\}$ and $(S, \bar{S})=$ $\{(i, j): i \in S, j \in \bar{S}\}=\{(1,2),(1,3)\}$. Thus, we have

$\overline{\tilde{\tilde{w}}}_{1}^{P} \oplus \tilde{\bar{c}}_{12}^{P}=\langle[0,0],[1,1]\rangle \oplus\langle[0.4,0.5],[0.3,0.4]\rangle$ $=\langle[0.4,0.5],[0.3,0.4]\rangle$

$\underline{\tilde{\tilde{w}}}_{1}^{P} \oplus \underline{\bar{c}}_{13}^{P}=\langle[0,0],[1,1]\rangle \oplus\langle[0.6,0.7],[0.2,0.3]\rangle$ $=\langle[0.6,0.7],[0.2,0.3]\rangle$

Therefore,

$M\left(\overline{\tilde{\tilde{w}}}_{1}^{P} \oplus \tilde{\bar{c}}_{12}^{P}\right)=M(\langle[0.4,0.5],[0.3,0.4]\rangle)=0.14$

$M\left(\overline{\tilde{\tilde{w}}}_{1}^{P} \oplus \underline{\tilde{\bar{c}}}_{13}^{P}\right)=M(\langle[0.6,0.7],[0.2,0.3]\rangle)=0.61$

Since $M\left(\underline{\tilde{\tilde{w}}}_{1}^{P} \oplus \underline{\tilde{\bar{c}}}_{12}^{P}\right)=\min \{0.14,0.61\}=0.14$, we set $\overline{\tilde{\tilde{w}}}_{2}^{P}=\overline{\tilde{\tilde{w}}}_{1}^{P} \oplus \tilde{\overline{\tilde{c}}}_{12}^{P}=\langle[0.4,0.5],[0.3,0.4]\rangle, \operatorname{Pred}\{2\}=1$, and $S=\{1,2\}$.

Iteration 2:

Let $\bar{S}=V-S=\{3,4,5,6\}$ and $(S, \bar{S})=$ $\{(i, j): i \in S, j \in \bar{S}\}=\{(1,3),(2,3),(2,4),(2,5)\}$. Thus, we have
$\underline{\tilde{\tilde{w}}}_{1}^{P} \oplus \underline{\tilde{\bar{c}}}_{13}^{P}=\langle[0,0],[1,1]\rangle \oplus\langle[0.6,0.7],[0.2,0.3]\rangle$

$$
=\langle[0.6,0.7],[0.2,0.3]\rangle
$$

$\overline{\tilde{w}}_{2}^{P} \oplus \tilde{\bar{c}}_{23}^{P}=\langle[0.4,0.5],[0.3,0.4]\rangle \oplus\langle[0.3,0.6],[0.3,0.4]\rangle$ $=\langle[0.48,0.72],[0.09,0.16]\rangle$

$\overline{\tilde{w}}_{2}^{P} \oplus \tilde{\bar{c}}_{24}^{P}=\langle[0.4,0.5],[0.3,0.4]\rangle \oplus\langle[0.7,0.8],[0.1,0.2]\rangle$ $=\langle[0.76,0.85],[0.03,0.08]\rangle$

$\overline{\tilde{\tilde{w}}}_{2}^{P} \oplus \tilde{\bar{c}}_{25}^{P}=\langle[0.4,0.5],[0.3,0.4]\rangle \oplus\langle[0.6,0.7],[0.2,0.3]\rangle$ $=\langle[0.68,0.78],[0.06,0.12]\rangle$

Therefore,

$M\left(\overline{\tilde{\tilde{w}}}_{1}^{P} \oplus \underline{\tilde{c}}_{13}^{P}\right)=M(\langle[0.6,0.7],[0.2,0.3]\rangle)=0.61$

$M\left(\overline{\tilde{\tilde{w}}}_{2}^{P} \oplus \underline{\tilde{\bar{c}}}_{23}^{P}\right)=M(\langle[0.48,0.72],[0.09,0.16]\rangle)=0.62$

$M\left(\overline{\tilde{\tilde{w}}}_{2}^{P} \oplus \underline{\tilde{\bar{c}}}_{24}^{P}\right)=M(\langle[0.76,0.85],[0.03,0.08]\rangle)=0.97$

$M\left(\overline{\tilde{w}}_{2}^{P} \oplus \underline{\bar{c}}_{25}^{P}\right)=M(\langle[0.68,0.78],[0.06,0.12]\rangle)=0.87$

Since $M\left(\overline{\tilde{w}}_{1}^{P} \oplus \underline{\bar{c}}_{13}^{P}\right)=\min \{0.61,0.62,0.99,0.87\}=$ 0.61 , then we set $\overline{\tilde{\tilde{w}}}_{3}^{P}=\underline{\tilde{\tilde{w}}}_{1}^{P} \oplus \underline{\tilde{\bar{c}}}_{13}^{P}=$ $\langle[0.6,0.7],[0.2,0.3]\rangle, \operatorname{Pred}\{3\}=1$, and $S=\{1,2,3\}$.

Iteration 3:

Let $\bar{S}=V-S=\{4,5,6\}$ and $(S, \bar{S})=$ $\{(i, j): i \in S, j \in \bar{S}\}=\{(2,4),(2,5),(3,4),(3,5)\}$. Thus, we have

$\overline{\tilde{w}}_{2}^{P} \oplus \tilde{\bar{c}}_{24}^{P}=\langle[0.4,0.5],[0.3,0.4]\rangle \oplus\langle[0.7,0.8],[0.1,0.2]\rangle$ $=\langle[0.76,0.85],[0.03,0.08]\rangle$

$\overline{\tilde{w}}_{2}^{P} \oplus \underline{\bar{c}}_{-25}^{P}=\langle[0.4,0.5],[0.3,0.4]\rangle \oplus\langle[0.6,0.7],[0.2,0.3]\rangle$ $=\langle[0.68,0.78],[0.06,0.12]\rangle$

$\overline{\tilde{\tilde{w}}}_{3}^{P} \oplus \underline{\tilde{\bar{c}}}_{34}^{P}=\langle[0.6,0.7],[0.2,0.3]\rangle \oplus\langle[0.4,0.6],[0.2,0.4]\rangle$ $=\langle[0.68,0.82],[0.04,0.12]\rangle$

$\overline{\tilde{w}}_{3}^{P} \oplus \tilde{\bar{c}}_{35}^{P}=\langle[0.6,0.7],[0.2,0.3]\rangle \oplus\langle[0.7,0.8],[0.3,0.5]\rangle$ $=\langle[0.82,0.90],[0.06,0.15]\rangle$

Therefore,

$M\left(\overline{\tilde{\tilde{w}}}_{2}^{P} \oplus \underline{\tilde{\bar{c}}}_{24}^{P}\right)=M(\langle[0.76,0.85],[0.03,0.08]\rangle)=0.97$ $M\left(\overline{\tilde{\tilde{w}}}_{2}^{P} \oplus \underline{\overline{\bar{c}}}_{25}^{P}\right)=M(\langle[0.68,0.78],[0.06,0.12]\rangle)=0.87$ 


$$
\begin{aligned}
& M\left(\overline{\tilde{\tilde{w}}}_{3}^{P} \oplus \stackrel{\tilde{\bar{c}}}{ }_{34}^{P}\right)=M(\langle[0.68,0.82],[0.04,0.12]\rangle)=0.91 \\
& M\left(\tilde{\overline{\tilde{w}}}_{3}^{P} \oplus \stackrel{\tilde{\bar{c}}}{ }_{35}^{P}\right)=M(\langle[0.82,0.90],[0.06,0.15]\rangle)=0.99
\end{aligned}
$$

Since $M\left(\overline{\tilde{w}}_{2}^{P} \oplus \underline{\bar{c}}_{25}^{P}\right)=\min \{0.97,0.87,0.91,0.99\}=$ 0.87 , we set $\overline{\tilde{w}}_{5}^{P}=\overline{\tilde{w}}_{2}^{P} \oplus \tilde{\bar{c}}^{P}=\langle[0.68,0.78]$, [0.06, 0.12] $\rangle$, $\operatorname{Pred}\{5\}=2$, and $S=\{1,2,3,5\}$.

Iteration 4:

Let $\bar{S}=V-S=\{4,6\}$ and $(S, \bar{S})=$ $\{(i, j): i \in S, j \in \bar{S}\}=\{(3,4),(5,6)\}$. Thus, we have

$$
\begin{aligned}
\underline{\tilde{\tilde{w}}}_{3}^{P} \oplus \underline{\overline{\tilde{c}}}_{34}^{P}= & \langle[0.6,0.7],[0.2,0.3]\rangle \\
& \oplus\langle[0.4,0.6],[0.2,0.4]\rangle \\
& =\langle[0.68,0.82],[0.04,0.12]\rangle \\
\overline{\tilde{\tilde{w}}}_{5}^{P} \oplus \underline{\tilde{\bar{c}}}^{P}= & \langle[0.68,0.78],[0.06,0.12]\rangle \\
& \oplus\langle[0.3,0.4],[0.1,0.2]\rangle \\
& =\langle[0.71,0.82],[0.018,0.024]\rangle
\end{aligned}
$$

Therefore,

$$
\begin{aligned}
& M\left(\overline{\tilde{w}}_{3}^{P} \oplus \underline{\tilde{\bar{c}}}_{34}^{P}\right)=M(\langle[0.68,0.82],[0.04,0.12]\rangle)=0.91 \\
& M\left(\overline{\tilde{\tilde{w}}}_{5}^{P} \oplus \stackrel{\tilde{\bar{c}}}{ }^{P}\right)=M(\langle[0.71,0.82],[0.018,0.024]\rangle)=0.957
\end{aligned}
$$

Since $M\left(\overline{\tilde{\tilde{w}}}_{3}^{P} \oplus \underline{\tilde{c}}^{P}\right)=\min \{0.91,0.95\}=0.91$, we set $\overline{\tilde{\tilde{w}}}_{4}^{P}=\overline{\tilde{\tilde{w}}}_{3}^{P} \oplus \tilde{\bar{c}}_{34}^{P}=\langle[0.68,0.82],[0.04,0.12]\rangle, \operatorname{Pred}\{4\}=$ 3 , and $S \stackrel{3}{=}\{1,2,3,5,4\}$.

Iteration 5:

Let $\bar{S}=V-S=\{6\}$ and $(S, \bar{S})=$ $\{(i, j): i \in S, j \in \bar{S}\}=\{(4,6),(5,6)\}$. Thus, we have

$$
\begin{aligned}
\underline{\tilde{\tilde{w}}}_{5}^{P} \oplus \underline{\tilde{\bar{c}}}^{P}= & \langle[0.68,0.78],[0.06,0.12]\rangle \\
& \oplus\langle[0.3,0.4],[0.1,0.2]\rangle \\
& =\langle[0.71,0.82],[0.018,0.024]\rangle \\
\underline{\tilde{w}}_{4}^{P} \oplus \underline{\tilde{c}}^{P}= & \langle[0.68,0.82],[0.04,0.12]\rangle \\
& \oplus\langle[0.4,0.7],[0.1,0.2]\rangle \\
= & \langle[0.74,0.91],[0.004,0.024]\rangle
\end{aligned}
$$

Therefore,

$$
M\left(\overline{\tilde{w}}_{5}^{P} \oplus \underline{\bar{c}}_{56}^{P}\right)=M(\langle[0.71,0.82],[0.018,0.024]\rangle)=0.95
$$

$M\left(\overline{\tilde{\tilde{w}}}_{4}^{P} \oplus \underline{\overline{\tilde{c}}}_{46}^{P}\right)=M(\langle[0.74,0.91],[0.004,0.024]\rangle)=0.99$

Since $\quad M\left(\overline{\tilde{\tilde{w}}}_{5}^{P} \oplus \underline{\overline{\tilde{c}}}_{56}^{P}\right)=\min \{0.95,0.99\}=0.95, \quad$ we set $\quad \overline{\tilde{w}}_{6}^{P}=\overline{\tilde{\tilde{w}}}_{5}^{P} \quad \oplus \quad \quad_{56}{ }^{P}=\langle[0.71,0.82],[0.018,0.024]\rangle$, $\operatorname{Pred}\{6\}=5$, and $S=\{1,2,3,5,4,6\}$.

This means that the IVPFSP cost from the source node 1 to node 6 is equal to $\overline{\tilde{w}}_{6}^{P}=\langle[0.71,0.82],[0.018,0.024]\rangle$. The corresponding IVPFSP can be found as follows:

$\operatorname{Pred}\{6\}=5, \operatorname{Pred}\{5\}=2, \operatorname{Pred}\{2\}=1$

Hence, the IVPF shortest path is $p_{16}: 1 \rightarrow 2 \rightarrow 5 \rightarrow 6$.

\section{Conclusions}

Traditional SP problem requires precise arc weights which is not always the case in real-life applications. In this present work, a shortest path problem having an intervalvalued Pythagorean fuzzy arc costs has been investigated. We first formulated the SP problem in the interval-valued Pythagorean fuzzy environment. We used an existing improved score function to compare the costs between different paths with their arc costs represented by IVPFNs. Based on this improved score function, we described the IVPF shortest path optimality conditions for the SP problem under consideration. The traditional Dijkstra's algorithm has been generalized to determine the IVPF cost of the shortest path and corresponding IVPFSP. Finally, a small sized telecommunication network has been provided to illustrate the proposed algorithm under IVPF environment. In the future, we will extend the method to more complicated network problems involving negative and non-negative IVPF costs. The proposed approach for solving SP problems in IVPF environment can be extended for solving them in generalized Pythagorean fuzzy environment [28, 29]. Moreover, he development of the proposed method for deriving the IVPF shortest path between all pairs of nodes is left to the next study.

Acknowledgement The authors would like to thank the anonymous reviewers and the editors for their insightful comments and suggestions.

Open Access This article is distributed under the terms of the Creative Commons Attribution 4.0 International License (http://creativecomm ons.org/licenses/by/4.0/), which permits unrestricted use, distribution, and reproduction in any medium, provided you give appropriate credit to the original author(s) and the source, provide a link to the Creative Commons license, and indicate if changes were made. 


\section{References}

1. Zadeh LA (1965) Fuzzy sets. Inf Control 8:338-353

2. Okada S, Super T (2000) A shortest path problem on a network with fuzzy arc lengths. Fuzzy Sets Syst 109:129-140

3. Keshavarz E, Khorram E (2009) A fuzzy shortest path with the highest reliability. J Comput Appl Math 230:204-212

4. Dou Y, Zhu L, Wang HS (2012) Solving the fuzzy shortest path problem using multi-criteria decision method based on vague similarity measure. Appl Soft Comput 12(6):1621-1631

5. Deng Y, Chen Y, Zhang Y, Mahadevan S (2012) Fuzzy Dijkstra algorithm for shortest path problem under uncertain environment. Appl Soft Comput 12:1231-1237

6. Ebrahimnejad A, Karimnejad Z, Alrezaamiri H (2015) Particle swarm optimization algorithm for solving shortest path problems with mixed fuzzy arc weights. Int J Appl Decis Sci 8(2):203-222

7. Ebrahimnejad A, Tavana M, Alrezaamiri H (2016) A novel artificial bee colony algorithm for shortest path problems with fuzzy arc weights. Measurement 93:48-56

8. Atanassov KT (1986) Intuitionistic fuzzy sets. Fuzzy Sets Syst 20:87-96

9. Mukherjee S (2012) Dijkstra's algorithm for solving the shortest path problem on networks under intuitionistic fuzzy environment. J Math Modell Algorithm 11(4):345-359

10. Geetharamani G, Jayagowri P (2012) Using similarity degree approach for shortest path in Intuitionistic fuzzy network, In: International conference on computing, communication and applications (ICCCA), IEEE, Dindigul, Tamilnadu, India, pp 1-6

11. Biswas SS, Bashir A, Doja MN (2013) An algorithm for extracting intuitionistic fuzzy shortest path in a graph. Appl Comput Intell Soft Comput 2013:1-5

12. Kumar G, Bajaj RK, Gandotra N (2015) Algorithm for shortest path problem in a network with interval-valued intuitionistic trapezoidal fuzzy number. Procedia Comput Sci 70:123-129

13. Sujatha L, Hyacinta JD (2017) The shortest path problem on networks with intuitionistic fuzzy edge weights. Glob J Pure Appl Math 13(7):3285-3300

14. Motameni H, Ebrahimnejad A (2018) Constraint shortest path problem in a network with intuitionistic fuzzy arc weights. In: Medina J, Ojeda-Aciego M, Verdegay J, Perfilieva I, BouchonMeunier B, Yager R (eds) Information processing and management of uncertainty in knowledge-based systems. Applications. IPMU 2018. Communications in computer and information science, vol 855. Springer, Cham

15. Yager RR (2014) Pythagorean membership grades in multicriteria decision making. IEEE Trans Fuzzy Syst 22:958-965

16. Zhang $X$ (2016) Multicriteria Pythagorean fuzzy decision analysis: a hierarchical QUAL-IFLEX approach with the closeness indexbased ranking methods. Inf Sci 330:104-124

17. Zhang XL, Xu ZS (2014) Extension of TOPSIS to multicriteria decision making with Pythagorean fuzzy sets. Int J Intell Syst 29:1061-1078
18. Garg H (2016) A novel accuracy function under interval-valued Pythagorean fuzzy environment for solving multicriteria decision making problem. J Intell Fuzzy Syst 31(1):529-540

19. Garg H (2018) A linear programming method based on an improved score function for interval-valued Pythagorean fuzzy numbers and its application to decision-making. Int J Uncertain Fuzz Knowl Based Syst 26(1):67-80

20. Garg H (2018) Generalized Pythagorean fuzzy geometric interactive aggregation operators using Einstein operations and their application to decision making. J Exp Theor Artif Intell. https://do i.org/10.1080/0952813x.2018.1467497

21. Garg H (2018) Linguistic Pythagorean fuzzy sets and its applications in multi attribute decision making process. Int $\mathbf{J}$ Intell Syst 33(6): 1234-1263

22. Garg H (2018) Hesitant Pythagorean fuzzy sets and their aggregation operators in multiple-attribute decision-making. Int J Uncertain Quantif 8(3):267-289

23. Garg H (2018) A new exponential operational laws and their aggregation operators of interval-valued Pythagorean fuzzy information. Int J Intell Syst 33(3):653-683

24. Garg H (2018) Some methods for strategic decision-making problems with immediate probabilities in Pythagorean fuzzy environment. Int J Intell Syst Wiley 33(4):687-712

25. Garg H (2017) Confidence levels based Pythagorean fuzzy aggregation operators and its application to decision-making process. Comput Math Organ Theory Springer 23(4):546-571

26. Garg H (2017) A novel improved accuracy function for interval valued Pythagorean fuzzy sets and its applications in decision making process. Int J Intell Syst 32(12):1247-1260

27. Garg H (2016) A novel correlation coefficients between Pythagorean fuzzy sets and its applications to decision-making processes. Int J Intell Syst 31(12):1234-1253

28. Garg H (2016) A new generalized Pythagorean fuzzy information aggregation using Einstein operations and its application to decision making. Int J Intell Syst 31(9):886-920

29. Garg H (2017) Generalized Pythagorean fuzzy Geometric aggregation operators using Einstein t-norm and t-conorm for multicriteria decision-making process. Int J Intell Syst 32(6):597-630

Publisher's Note Springer Nature remains neutral with regard to jurisdictional claims in published maps and institutional affiliations 